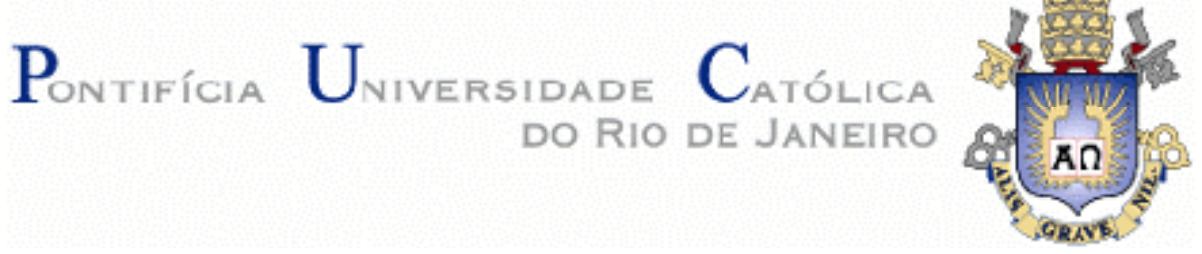

Gabriel Evangelista Medeiros

Síntese de Dissulfeto de Ferro para Aplicação em Pilhas

Térmicas

Dissertação de Mestrado

Dissertação apresentada como requisito parcial para obtenção do título de Mestre pelo Programa de Pós-Graduação em Engenharia de Materiais e de Processos Químicos e Metalúrgicos da PUCRio.

Orientador: Prof. Francisco José Moura

Rio de Janeiro

Abril de 2011 


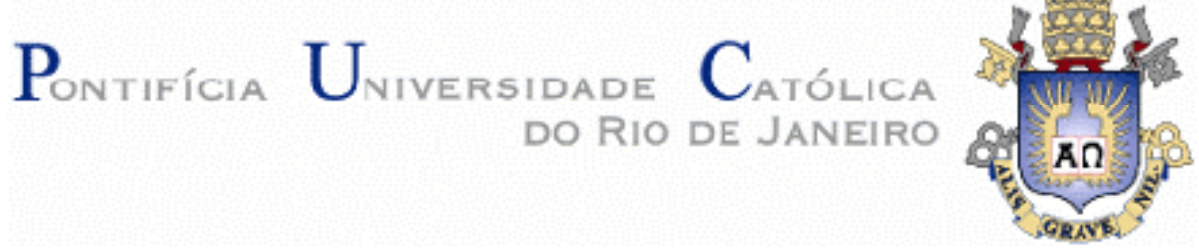

Gabriel Evangelista Medeiros

\section{Síntese de Dissulfeto de Ferro para Aplicação em Pilhas}

Térmicas

Dissertação apresentada como requisito parcial para obtenção do título de Mestre pelo Programa de PósGraduação em Engenharia de Materiais e de Processos Químicos e Metalúrgicos da PUC-Rio. Aprovada pela Comissão Examinadora abaixo assinada.

Prof. Francisco José Moura

Orientador

Departamento de Engenharia de Materiais - PUC-Rio

Prof. Maria José Panichi Vieira

Co-orientadora

Centro Tecnológico do Exército - CTEx

Prof. Eduardo de Albuquerque Brocchi Departamento de Engenharia de Materiais - PUC-Rio

Prof. Mirna Tamiozzo da Costa Rupp

Centro Tecnológico do Exército - CTEx

Prof. José Eugênio Leal

Coordenador Setorial do Centro Técnico Científico - PUC-Rio

Rio de Janeiro, 13 de abril de 2011 
Todos os direitos reservados. É proibida a reprodução total ou parcial do trabalho sem autorização da universidade, do autor e do orientador.

\section{Gabriel Evangelista Medeiros}

Graduou-se em Engenharia de Materiais na PUC-Rio (Pontifícia Universidade Católica do Rio de Janeiro) em 2008. Atuou, durante o desenvolvimento de seu trabalho de mestrado, no Laboratório de Química Militar do Centro Tecnológico do Exército (CTEx). Fez iniciação científica em processamento de resíduos de biomassa para geração de substâncias húmicas com aplicações de Engenharia Ambiental. Atua no ramo de siderurgia desde 2010.

Ficha Catalográfica

Medeiros, Gabriel Evangelista

Síntese de dissulfeto de ferro para aplicação em pilhas térmicas / Gabriel Evangelista Medeiros; orientador: Francisco José Moura. - PUC, Departamento de Engenharia de Materiais, 2011.

85 f. :il. ; $30 \mathrm{~cm}$

Dissertação (mestrado) - Pontifícia Universidade Católica do Rio de Janeiro, Departamento de Engenharia de Materiais, 2011.

Inclui referências bibliográficas.

1. Engenharia de materiais - Teses. 2. Dissulfeto de ferro $\left(\mathrm{FeS}_{2}\right)$. 3. Pilhas térmicas. 4. Cinética de sulfetação. 5. Óxido férrico. 6. Síntese em leito fluidizado. I. Moura, Francisco José. II. Vieira, Maria José Panichi. III. Pontifícia Universidade Católica do Rio de Janeiro. Departamento de Engenharia de Materiais. IV. Título.

CDD: 620.11 
Para minha mãe Neuza Lucia Evangelista Medeiros, meu pai Celso Cerqueira de Castro Medeiros, minha irmã Sabrina Evangelista Medeiros, meu cunhado Elmo Gomes Cavalcante Júnior e minha esposa Katia Maria dos Santos Calegaro, por todo apoio e confiança. 


\section{Agradecimentos}

A Deus, por me orientar e proporcionar força para vencer todos os desafios.

Aos orientadores Francisco José Moura e Maria José Panichi Vieira pelo estímulo, empenho e colaboração na realização deste trabalho.

À equipe do CTEx, por toda a colaboração e assistência prestada.

Aos meus pais, pelo apoio, compreensão, carinho e dedicação, desde os meus primeiros passos na vida.

À minha irmã, por acreditar sempre no meu potencial.

À minha esposa e amiga Katia Maria dos Santos Calegaro, pela paciência, amparo e compreensão em todos os momentos.

À PUC-Rio, pelos auxílios concedidos, sem os quais este trabalho não poderia ter sido realizado.

Ao CBPF, pelos auxílios concedidos, imprescindíveis à realização deste trabalho.

Ao meu amigo Rodrigo Fernandes Magalhães de Souza, pela cooperação e prestatividade nas análises de MEV.

Aos professores que participaram da banca examinadora pelo conhecimento compartilhado.

Aos meus colegas da PUC-Rio.

Aos professores do DEMA, especialmente Roberto Ribeiro de Avillez e Eduardo de Albuquerque Brocchi, que desde a graduação se empenharam em oferecer a mim as melhores oportunidades. 


\section{Resumo}

Medeiros, Gabriel Evangelista; Moura, Francisco José. Síntese de Dissulfeto de Ferro para Aplicação em Pilhas Térmicas. Rio de Janeiro, 2011. 85p. Dissertação de Mestrado - Departamento de Engenharia de Materiais, Pontifícia Universidade Católica do Rio de Janeiro.

A evolução tecnológica e a necessidade de fontes de energia cada vez mais eficientes e compactas alavancam os estudos eletroquímicos, no intuito de desenvolver fontes primárias e secundárias mais duráveis e que suportem uma maior carga de operação. Dentre as fontes eletroquímicas temos as primárias (pilhas) e as secundárias (baterias). A diferença fundamental entre essas fontes é o fato das baterias suportarem um número definido de ciclos de carga-descarga, enquanto as pilhas sofrem um único ciclo de descarga após o qual devem ser descartadas. É importante notar que a fonte deve ser adequada à aplicação para qual foi desenvolvida. Assim, em alguns casos especiais, como sistemas de emergência, aparatos militares e aeroespaciais, são necessárias fontes que possuam longa vida de prateleira, alta confiabilidade e alta densidade de corrente. Nesse contexto, enquadram-se as pilhas térmicas que são fontes primárias, nãorecarregáveis e inertes à temperatura ambiente. No desenvolvimento das pilhas térmicas o sistema de última geração funciona com anodos de liga de Lítio, eletrólitos eutéticos de $\mathrm{LiCl}-\mathrm{KCl}$ e catodos de $\mathrm{FeS}_{2}$. O objetivo deste trabalho foi estudar uma rota de síntese pirometalúrgica a partir da reação de ustulação sulfetante do $\mathrm{Fe}_{2} \mathrm{O}_{3}$ com enxofre vaporizado, para obtenção do $\mathrm{FeS}_{2}$ de alta pureza, utilizado como despolarizante nas pilhas. As variáveis de estudo foram a temperatura e o tempo de reação, além da temperatura de volatilização do enxofre e, para avaliação dos resultados foram realizadas caracterizações dos produtos em MEV e DRX, com auxílio do método quantitativo de Rietveld. Os resultados obtidos mostraram uma conversão próxima de 90 \% e uma alta dispersão de tamanho de partículas devido à agregação das mesmas. Todavia, a desagregação manual pode resultar em partículas menores adequadas à fabricação do catodo.

\section{Palavras-chave}

Dissulfeto de ferro (FeS2); pilhas térmicas; cinética de sulfetação; óxido férrico; síntese em leito fluidizado. 


\section{Abstract}

Medeiros, Gabriel Evangelista; Moura, Francisco José (Advisor). Sinthesis of Iron Disulphide for Thermal Batteries Aplication. Rio de Janeiro, 2011. 85p. MSc. Dissertation - Departamento de Engenharia de Materiais, Pontifícia Universidade Católica do Rio de Janeiro.

The continuous evolution of new technologies and the requirement for more efficient and compact power sources, justify many electrochemical researches in order to develop new types of primary and secondary power sources achieving longer operational lives and more density of energy. The primary power sources are not capable of running on charges-discharges cycles and because of this have to be discarded after the ending of electrochemical reactions (end of operational life). On the other hand the secondary sources are capable of doing charges cycles and therefore have a longer life. It is very important to realize that each kind of power source should be proper to specific applications. Though, in some cases, like emergency systems, military equipments and air and space features, it is necessary to have long shelf life sources, high reliability and high density of energy. For these special applications some sources called thermal batteries fit very well and these are primary electrochemical sources, non-rechargeable and inert at the room temperature. On the development of thermal batteries there was consolidated the technology that uses lithium-alloy anodes, eutectic salt electrolytes based on $\mathrm{LiCl}-\mathrm{KCl}$ and $\mathrm{FeS}_{2}$ cathodes. The main goal of this work is to study a pyrometallugical route for the synthesis of high purity $\mathrm{FeS}_{2}$ by the reaction of vaporized sulfur and $\mathrm{Fe}_{2} \mathrm{O}_{3}$, for further constructions of thermal batteries prototypes. The variables of the study are temperature and time of reaction, besides the volatilizing temperature of sulfur. The analysis was done by MEV and XRD within the Rietveld method. The results showed almost $90 \%$ but a high dispersion of particles sizes. It is expected to obtain less dispersion of sizes by disaggregation methods.

\section{Keywords}

Iron disulphide (FeS2); thermal batteries; kinetics of sulphidation; iron oxide; fluidized bath synthesis. 


\section{Sumário}

1 INTRODUÇÃO

2 REVISÃO BIBLIOGRÁFICA 16

2.1. Fontes Eletroquímicas 16

2.1.1. Fontes Primárias Comuns 17

2.1.2. Pilhas Térmicas 18

2.2. Obtenção do Dissulfeto de Ferro 23

2.2.1. Purificação Mineral 24

2.2.2. Síntese Hidrometalúrgica 26

2.2.3. Rota Pirometalúrgica $\quad 29$

3 FUNDAMENTOS TEÓRICOS 32

3.1. Avaliação Termodinâmica 32

3.2. Reações em Leito Fluidizado 40

3.2.1. Fluidização Agregativa 44

3.2.2. Diagrama de Grace 46

4 METODOLOGIA EXPERIMENTAL 49

4.1. As Matérias-Primas $\quad 49$

4.2. A Linha de Síntese 49

4.2.1. Procedimento Experimental da Síntese 55

4.3. Análise das Amostras 58

4.3.1. Difração de Raios-X 58

4.3.2. Microscopia Eletrônica de Varredura 59

5 RESULTADOS E DISCUSSÃO 60

5.1. Avaliação do Efeito das Variáveis Operacionais na Conversão $\quad 60$

5.1.1. Lavagem do Produto da Reação 62

5.2. Caracterização dos Produtos $\quad 67$

5.3. Avaliação Fluidodinâmica 77 
6 Conclusão $\quad 80$

7 Sugestões para Outros Trabalhos 81

8 Referências Bibliográficas 83 


\section{Lista de Figuras}

Figura 2.1 - Forma e variação de dimensões das pilhas térmicas [4].

Figura 2.2 - Figura esquemática dos componentes internos de uma

pilha de dissulfeto de ferro [4].

Figura 2.3 - Pilha térmica construída num pequeno volume com uma f.e.m. de 500V [4].

Figura 2.4 - Diagrama de fases binário Li-Al [11]. 22

Figura 2.5 - Diagrama binário de equilíbrio do sistema Li-Si [8]. 23

Figura 2.6 - Ocorrência natural de macrocristais de pirita [14]. 24

Figura 2.7 - Rota de purificação aplicada por Amorim et al [16]. 26

Figura 2.8 - Morfologia da pirita precipitada a partir de FeS e $\mathrm{H}_{2} \mathrm{~S}$

$(\mathrm{pH}=6$ e $\mathrm{Eh}=250 \mathrm{mV})[21] . \quad 29$

Figura 3.1 - Diagrama de distribuição de espécies de enxofre gasoso

numa atmosfera de argônio [18].

Figura 3.2 - Pressão de vapor de enxofre total em função da temperatura [16]. 34

Figura 3.3 - Diagrama de predominância do sistema Fe-S-O para

temperatura de $300^{\circ} \mathrm{C}[18]$.

Figura 3.4 - Diagrama de predominância do sistema Fe-S-O para temperatura de $400^{\circ} \mathrm{C}[18]$.

Figura 3.5 - Diagrama de predominância do sistema Fe-S-O para temperatura de $500^{\circ} \mathrm{C}[18]$.

Figura 3.6 - Diagrama de predominância do sistema Fe-S-O para temperatura de $600^{\circ} \mathrm{C}[18]$.

Figura 3.7 - Diagrama de predominância do sistema Fe-S-O para

$\mathrm{pO}_{2}=0,21$ atm [18].

Figura 3.8 - Diagrama de predominância do sistema Fe-S-O para $\mathrm{pO}_{2}=10^{-20}$ atm [18].

Figura 3.9 - Esquema da distribuição de forças atuando sobre uma partícula num fluxo gasoso.

Figura 3.10 - Perda de carga do gás em função da velocidade num modelo de leito fluidizado. 
Figura 3.11 - Diferentes regimes de fluidização em função da

velocidade do fluido [24].

Figura 3.12. Diagrama de Grace, ilustrando as regiões de diferentes

modos (agregativo e particulado) e regimes de fluidização

(leito fixo, pistonamento e regime turbulhento) [24].

Figura 4.1 - Linha de síntese utilizada para obtenção da pirita.

Figura 4.2 - Bancada móvel com o conjunto reator e volatilizador

e caixa controladora.

Figura 4.3 - Destaque para o controlador de temperatura e entrada de gás inerte para o volatilizador.

Figura 4.4 - Destaque para o forno e saída de gás do reator.

Figura 4.5 - Reator e volatilizador desmontados, onde se destaca

a resistência elétrica de imersão, e os componentes do reator.

Figura 4.6 - Vista superior interna do reator, destacando o alargamento

de seção e a placa porosa.

Figura 4.7 - Fluxograma das principais etapas do procedimento experimental. 56

Figura 4.8 - Avaliação da taxa de aquecimento do reator com e sem

o isolamento térmico adequado.

Figura 5.1 - Conversão final (\%) vs tempo de reação, para diferentes

condições, onde Tr é a temperatura de reação e Tv é a temperatura

de volatilização de enxofre.

Figura 5.2 - DRX da amostra 1, após lavagem com HCl concentrado e $\mathrm{CS}_{2} .63$

Figura 5.3 - MEV a 400x da amostra 1 após lavagem. 63

Figura 5.4 - MEV a 1800x da amostra 1 lavada. 64

Figura 5.5 - DRX da amostra 2 após lavagem. $\quad 64$

Figura 5.6 - MEV a 400x da amostra 2 lavada. $\quad 65$

Figura 5.7 - MEV a 1800x da amostra 2 após lavagem. 66

Figura 5.8 - Imagem de fotografia digital de uma amostra a $500^{\circ} \mathrm{C}$

com volatilização do enxofre a $250^{\circ} \mathrm{C}$ e 1 hora de reação, ilustrando

dispersão de tamanho de partículas e núcleos não reagidos.

Figura 5.9 - MEV da corrida D em 400x, utilizado para varredura de EDS. 68

Figura 5.10 - MEV da corrida D em 1800x. 68

Figura 5.11 - Difração de Raios-X da Corrida D. $\quad 69$ 
Figura 5.12 - MEV da corrida A em 400x utilizada para varredura do EDS. $\quad 70$

Figura 5.13 - MEV da corrida A em 1800x. 70

Figura 5.14 - Difração de Raios-X da Corrida A com análise quantitativa. $\quad 71$

Figura 5.15 - MEV da corrida C em 400x utilizada para varredura de EDS. 72

Figura 5.16 - MEV da corrida C em 1800x. 72

Figura 5.17 - Difração de Raios-X da corrida C com análise quantitativa. $\quad 73$

Figura 5.18 - MEV da corrida G em 400x utilizada para varredura de EDS. $\quad 74$

Figura 5.19 - MEV da corrida G em 1800x. 74

Figura 5.20 - Difração de Raios-X da corrida G com análise quantitativa. $\quad 75$

Figura 5.21 - MEV da corrida H em 400x utilizada para a varredura de EDS. 76

Figura 5.22 - MEV da corrida H em 1800x. 76

Figura 5.23 - Difração de Raios-X da corrida H com análise quantitativa. $\quad 77$

Figura 5.24. Diagrama de Grace de acordo com as condições experimentais

$\begin{array}{ll}\text { reais. } & 79\end{array}$ 


\section{Lista de Tabelas}

Tabela 2.1 - Dados termodinâmicos da reação de precipitação da pirita $\begin{array}{ll}\text { em solução de ácido sulfídrico [18]. } & 27\end{array}$

Tabela 3.1 - Dados termodinâmicos para a reação de $\mathrm{Fe}_{2} \mathrm{O}_{3}$ e $\mathrm{S}_{2(\mathrm{~g})}[18]$.

Tabela 3.2. Relações experimentais para a velocidade mínima de fluidização [24].

Tabela 3.3. Expressões para determinação da velocidade mínima de fluidização em regime borbulhante [24].

Tabela 3.4. Expressões para determinação da velocidade mínima de pistonamento [24].

Tabela 3.5. Expressões para determinação da velocidade mínima de transporte pneumático [24]. $\quad 46$

Tabela 5.1 - Corridas analisadas e variáveis operacionais utilizadas. 